EPJ Web of Conferences 19, 03009 (2012)

DOI: $10.1051 /$ epjconf/20121903009

(C) Owned by the authors, published by EDP Sciences, 2012

\title{
Schwarzschild models of the Sculptor dSph galaxy
}

\author{
M.A. Breddels ${ }^{1, a}$, A. Helmi ${ }^{1}$, R.C.E. van den Bosch², G. van de Ven² \\ and G. Battaglia ${ }^{3}$
}

${ }^{1}$ Kapteyn Astronomical Institute, University of Groningen, PO Box 800, 9700 AV Groningen, The Netherlands

${ }^{2}$ Max-Planck-Institute for Astronomy, Königstuhl 17, 69117 Heidelberg, Germany

${ }^{3}$ European Organisation for Astronomical Research in the Southern Hemisphere, K. Schwarzschild-Str. 2, 85748 Garching bei München, Germany

\begin{abstract}
We have developed a spherically symmetric dynamical model of a dwarf spheroidal galaxy using the Schwarzschild method. This type of modelling yields constraints both on the total mass distribution (e.g. enclosed mass and scale radius) as well as on the orbital structure of the system modelled (e.g. velocity anisotropy). Therefore not only can we derive the dark matter content of these systems, but also explore possible formation scenarios. Here we present preliminary results for the Sculptor dSph. We find that the mass of Sculptor within $1 \mathrm{kpc}$ is $8.5 \times 10^{7 \pm 0.05} M_{\odot}$, its anisotropy profile is tangentially biased and slightly more isotropic near the center. For an NFW profile, the preferred concentration $(\sim 15)$ is compatible with cosmological models. Very cuspy density profiles (steeper than NFW) are strongly disfavoured for Sculptor.
\end{abstract}

\section{INTRODUCTION}

The existence of dark matter has been invoked to explain discrepancies in the observed kinematics of (systems of) galaxies. Dwarf spheroidal galaxies appear to be one of the most dark matter dominated galaxies, with total dynamical mass to stellar light ratios in the order of $100-1000 M_{\odot} / L_{\odot}$. The nearby dSph galaxies have the additional advantage that individual stars can be resolved, and their red giant branch stars are bright enough to measure line-of-sight velocities with errors of a few $\mathrm{km} \mathrm{s}^{-1}$. Their high dynamical mass-to-light ratios makes these systems ideal to study dark matter halos, especially their internal structure and to constrain their inner density profiles. To this end we study the Sculptor dwarf spheroidal galaxy using orbit based dynamical models, also known as Schwarzschild models.

\section{METHOD AND DATA}

In our Schwarzschild models, we assume a specific gravitational potential in which we integrate test particle orbits. These orbits are drawn from a dense grid in the space of integrals of motion (in our case energy and angular momentum). Using quadratic programming we find the set of weights such that the superposition of the weighted orbits matches the data. In our case we fit the light distribution, which we assume to be a Plummer sphere, and the velocity dispersion profile. For Sculptor we use the 2304

\footnotetext{
ae-mail: breddels@astro.rug.nl
}

This is an Open Access article distributed under the terms of the Creative Commons Attribution-Noncommercial License 3.0, which permits unrestricted use, distribution, and reproduction in any noncommercial medium, provided the original work is properly cited. 

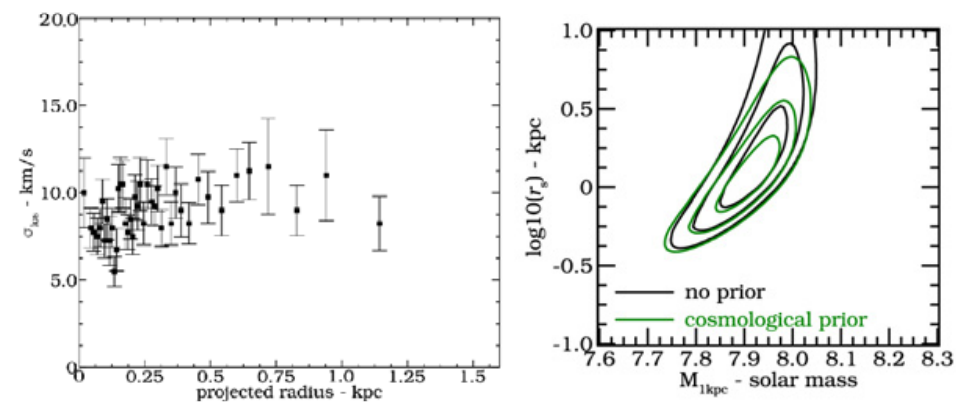

Figure 1. Left: Velocity dispersion profile for Sculptor. Right: Joint probability density functions for the enclosed mass within $1 \mathrm{kpc}$ and the NFW scale parameter $\left(r_{s}\right)$ for Sculptor.
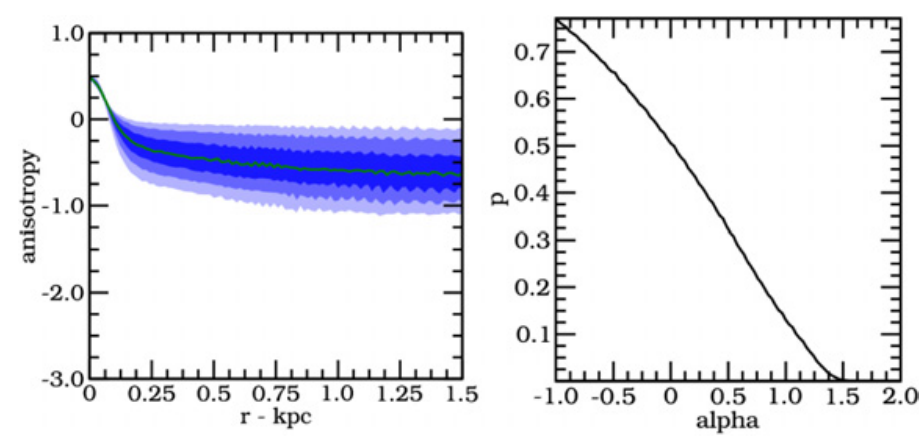

Figure 2. Left: Resulting anisotropy profiles for Sculptor. Right: Probability density function of the inner slope of the dark matter density profile for Sculptor.

line of sight velocities from the datasets by [1] and [2]. In the left panel of Fig. 1 we show the velocity dispersion profile we fit.

\section{RESULTS}

In the right panel of Fig. 1 we show the constraints on the mass and scale radius $\left(r_{s}\right)$ in the case an assumed NFW [4] profile for Sculptor. The black contours show the 1,2 and $3 \sigma$ confidence interval. It shows that although $\mathrm{M}(\mathrm{r}<1 \mathrm{kpc})$ is well constrained, the scale parameter $\left(r_{s}\right)$ is less so. The concentration $\left(r_{200} / r_{s} \approx 15\right)$ implied is compatible with cosmological models. The green contour includes a cosmological prior (the mass-concentration relation from [3]), which gives a slightly better constraint on $r_{s}$. Since the method finds the distribution function, we can also reconstruct the orbital structure. In the left panel of Fig 2 we show the anisotropy profile in the left panel of Fig. 2. The green line shows the median value, and the blue regions the 1,2,3 $\sigma$ confidence intervals. The velocity ellipsoid of Sculptor is more isotropic in the center compared to the outer regions. We also change the inner slope $(\alpha)$ of the DM density distribution $\rho(r)=\rho_{0}\left(r / r_{s}\right)^{-\alpha}\left(1+r / r_{s}\right)^{-2}$ (for NFW, $\alpha=1$ ). The probability density function of $\alpha$ is shown in the right panel of Fig. 2. Although the slope is not well constrained, very cuspy profiles $(\alpha>1.5)$ seem to be ruled out for Sculptor. More results can be found in [5]. 
Assembling the Puzzle of the Milky Way

\section{References}

[1] Battaglia, G. and Helmi, A. and Tolstoy, E. and Irwin, M. and Hill, V. and Jablonka, P., ApJ, 681, (2008) L13-L16

[2] Walker, M. G. and Mateo, M. and Olszewski, E. W., AJ, 137, (2009) 3100-3108

[3] Macciò, A. V. and Dutton, A. A. and van den Bosch, F. C. and Moore, B. and Potter, D. and Stadel, J., MNRAS, 378, (2007) 55-71

[4] Navarro, J. F. and Frenk, C. S. and White, S. D. M., ApJ, 462, (1996) $563-+$

[5] Breddels, M. A. and Helmi, A. and van den Bosch, R. C. E. and van de Ven, G. and Battaglia, G., MNRAS, (2011) submitted 